Revue Française de Civilisation Britannique

\title{
Civilisation et interdisciplinarité : Un entretien avec Jean-Paul Revauger
}

Civilization and Interdisciplinarity: an Interview with Jean-Paul Revauger

\section{Romain Garbaye et Gilles Leydier}

\section{(2) OpenEdition}

Journals

Édition électronique

URL : http://journals.openedition.org/rfcb/2982

DOI : $10.4000 /$ rfcb.2982

ISSN : 2429-4373

Éditeur

CRECIB - Centre de recherche et d'études en civilisation britannique

Référence électronique

Romain Garbaye et Gilles Leydier, « Civilisation et interdisciplinarité : Un entretien avec Jean-Paul Revauger », Revue Française de Civilisation Britannique [En ligne], XXIV-1 | 2019, mis en ligne le 22 mars 2019, consulté le 19 avril 2019. URL : http://journals.openedition.org/rfcb/2982 ; DOI : 10.4000/ rfcb.2982

Ce document a été généré automatiquement le 19 avril 2019

\section{(i) $\$$}

Revue française de civilisation britannique est mis à disposition selon les termes de la licence Creative Commons Attribution - Pas d'Utilisation Commerciale - Pas de Modification 4.0 International. 


\section{Civilisation et interdisciplinarité : Un entretien avec Jean-Paul Revauger}

Civilization and Interdisciplinarity: an Interview with Jean-Paul Revauger

Romain Garbaye et Gilles Leydier

Jean-Paul Revauger est professeur émérite de

civilisation britannique à l'Université Bordeaux

Montaigne, et ancien président du CRECIB.

\section{QU : Quel regard peut-on porter sur la civilisation britannique en tant que discipline de recherche au sein de l' « anglistique "? Et d'autre part comment envisager la relation entre " civilisation » et « interdisciplinarité »?}

1 Nous sommes en France, et nous nous situons dans une institution, l'université. Les réponses à vos questions sont nécessairement liées au contexte institutionnel dans lequel la discipline fonctionne.

2 Avant 1977, il y avait au sein de certains départements d'anglais des enseignements de civilisation, liés à l'esthétique, l'histoire des idées, l'histoire. Les recherches étaient rares, et le fait d'individus parfois brillants, mais en décalage par rapport à l' " anglistique ", où la littérature régnait sans partage.

En 1977, Monica Charlot parvint à faire créer une option civilisation à l'agrégation. Il n'y avait toujours pas de civilisation dans le tronc commun, mais c'était un début. Parallèlement, elle encouragea la création du $\mathrm{Crecib}^{1}$. L'orientation de Monica Charlot était proche des sciences politiques, et la plupart de ses doctorants et de ses soutiens s'inscrivaient dans cette optique. Roland Marx, angliciste historien, était moins influent, 
sur le plan institutionnel, malgré ses nombreuses publications. Cette situation perdura une dizaine d'années, avant que le Crecib accueille des chercheurs ayant d'autres démarches.

\section{QU : Comment se sont développées des dynamiques de recherche collective dans le domaine de la civilisation?}

4 A partir du début des années 80 , une dynamique se créa autour de François Poirier, de Paris VIII, et de Jean Pierre Ravier, angliciste de formation juridique, à Chambéry. L'orientation était différente, y compris sur le plan idéologique. Les thématiques étaient souvent liées à l'histoire du mouvement ouvrier, et aux mouvements hostiles au thatchérisme. Il y eut donc pendant plusieurs années une sorte de saine concurrence entre les deux foyers.

5 A partir du milieu des années 80 , la politique du Ministère changea, et il commença à soutenir fortement la recherche collective, ce qui modifia peu à peu la culture de toute l'université. Les études littéraires, jusqu'alors totalement individuelles, furent obligées de se structurer et d'adopter des logiques collectives. Les réseaux de recherche furent favorisés.

6 C'est à partir des années 90 que l'observatoire ${ }^{2}$ se mit en place, avec les universités de Grenoble III, Clermont Ferrand (avec Jacques Carré) et Lyon. Dans toutes les universités, la civilisation fonctionnait, selon les cas, soit en autonomie, soit au sein de centres de recherches anglicistes, autour de thématiques littéraires peu adaptées à la civilisation. Les relations n'étaient pas faciles avec les littéraires. D'un côté la civilisation était indispensable si on voulait des reçus à l'agrégation, de l'autre elle était perçue comme un nouveau venu un peu vulgaire.

7 La situation se mit à varier considérablement d'une université à une autre. Dans certains cas, l'histoire des idées et la civilisation se séparèrent, souvent pour des raisons personnelles, ailleurs, elles étaient indissociables. Dans d'autres, l'esthétique était très liée à l'étude du contexte historique, ailleurs elle restait dans l'abstraction, et plus proche de la littérature. Certains secteurs (dix huitiémistes, études écossaises) ne souhaitèrent pas séparer littérature et civilisation, car le mouvement des idées pour les uns, l'identité écossaise pour les autres étaient omniprésents dans les œuvres littéraires. Ces dernières avaient par ailleurs parfois un impact important sur la société.

\section{QU : Quel rapport aux cultural studies?}

La relation avec les cultural studies fut étudiée au début des années 90 (colloque de Grenoble en 1992). C'était d'autant plus tardif que, laminées par dix ans de thatchérisme, les cultural studies britanniques étaient à bout de souffle et se diversifiaient alors en gender studies, media studies ou post colonial studies, qui prirent leur autonomie à ce moment-là en Grande Bretagne. Certains civilisationnistes étaient hostiles aux cultural studies, soit pour des raisons idéologiques, soit par crainte d'un réinvestissement du champ de la civilisation par des littéraires déguisés. Cette problématique apparait toujours au moment des nominations sur poste. 
9 En tout cas le débat culture/civilisation, qui dure depuis le $19^{\text {ème }}$, permit la rédaction de belles dissertations.

10 A partir des années 90/2000 la civilisation trouva sa vitesse de croisière : des cours sont proposés depuis cette époque à tous les niveaux, y compris à l'agrégation, et un flux de publications et de thèses commença à alimenter la recherche. L'évaluation des travaux... et des chercheurs devint l'œuvre de la discipline elle-même, ce qui est une preuve de maturité. Le seul point négatif dans la situation fut la volonté la plus en plus nette du Ministère de regrouper chaque discipline dans un centre de recherches unique, dans chaque université. Se retrouvèrent donc dans le même chaudron littéraires, civilisationnistes, voire linguistes, forcés de définir des thématiques totalement artificielles, dont le libellé fait sourire ceux-là même qui les inventent.

\section{QU : Quelles relations avec les sciences humaines et sociales?}

11 Le rapport avec les sciences humaines ou sociales est resté une démarche individuelle. Très peu de civilisationnistes travaillent de façon régulière avec des centres autres qu'anglicistes, même s'il y a de vaillantes exceptions. Les difficultés objectives sont réelles. Certains milieux, comme celui des historiens, pratiquent une endogamie et un protectionnisme qui rendent impossible à un outsider formé en dehors du sérail d'obtenir une reconnaissance, à quelques exceptions près. La mentalité de forteresse assiégée qui s'est répandue dans l'université française depuis les crises liées à la mastérisation et la réforme du Capes touche à la fois les littéraires et les historiens. L'évolution des pratiques pédagogiques ne fait que renforcer le ressentiment. La relative dévalorisation de la transmission et de l'évaluation des connaissances, le discrédit des cours magistraux ont donné à beaucoup la conviction qu'il fallait se replier sur le noyau dur des disciplines et sur le classicisme, sans s'ouvrir à quoi que soit. Cela dit, les travaux de civilisationnistes à orientation historique sont bien sûr plus que bienvenus chez les anglicistes, et peuvent, au fond, parfaitement répondre aux normes en matière de sources secondaires et surtout primaires, de maîtrise du contexte et de problématisation. L'histoire du monde anglophone est d'autant moins une chasse gardée, que ce dernier est globalement très peu étudié par les historiens français, ne serait-ce que pour des raisons de maîtrise de la langue et de la culture.

12 En revanche, d'autres secteurs en pleine expansion, comme les sciences politiques sont nettement plus ouverts, tout en étant aussi farouchement compétitifs.

\section{QU : Quelles peuvent être les références théoriques communes aux civilisationnistes?}

La place de la théorie est bien sûr plus importante que ce n'était le cas traditionnellement en civilisation, et la difficulté pour les chercheurs civilisationnistes est de trouver où il faut placer le curseur dans ce domaine. Piller les manuels de sciences politique et plaquer des concepts abscons sur des recherches en réalité pragmatiques ne trompe personne, sauf les malheureux auteurs du forfait. Si on fait référence à un concept, encore faut-il en démontrer la pertinence. En revanche, il serait bon que les civilisationnistes débattent entre eux des structures théoriques qui leurs paraissent utiles dans leur champ, afin que 
se crée un corpus commun, au moins à une partie des civilisationnistes. Il faudrait bien sûr éviter de tomber dans les querelles de chapelle qui ont égayé la linguistique pendant des lustres. Mais un élément de théorisation permettrait de donner un peu plus de cohérence à notre discipline. C'est à ce prix que nous pourrons vraiment parler de transdisciplinarité.

\section{QU : Quelle place pour le comparatisme?}

14 Autre élément important dans une stratégie de rapprochement avec les sciences politiques, voire la sociologie, les approches comparatives. Les anglicistes sont certes les mieux placés pour travailler sur le monde anglophone, mais ils parlent à partir d'un contexte français, ce dont ils ont intérêt à être conscients. Leur regard n'est pas le même que celui d'un chercheur non francophone. Les sciences politiques, en particulier quand elles se laissent aller à une approche prescriptive, sont conscientes des écarts ou des similitudes entre la France et autres pays. L'angliciste serait donc bien avisé d'acquérir pour le moins une bonne connaissance des champs français équivalents à ceux qu'il étudie dans le monde anglophone. Dans ses échanges avec le milieu de la recherche hors de France, cela sera attendu de lui systématiquement.

\section{QU : Quelles perspectives pour le champ de la civilisation?}

Les chantiers qui sont devant la discipline sont enthousiasmants, car ils nous permettent d'évoluer, et de pousser plus avant notre curiosité intellectuelle. Une discipline sûre de ses acquis et fermée au changement est stérile. Le terme "civilisation », retenu par les Français pour décrire notre démarche, a l'avantage d'être totalement inclusif: les recherches à dominante politique, historique, esthétique, philosophique, économique peuvent parfaitement s'y reconnaitre, ce qui est un enjeu fondamental. Se fondre dans les autres secteurs des SHS reviendrait à nous priver de notre atout : une connaissance fine de la langue et des mentalités du monde anglophone, sans aucun doute plus approfondie que celle des non anglicistes. Par ailleurs, le terme "civilisation » a une signification particulière dans notre pays, où résonne le lointain écho des débats franco germaniques sur les caractéristiques spécifiques de la «civilisation française » et de la «Kultur » germanique. Ce n'est pas un hasard si le terme a les faveurs de bien des historiens. Malgré notre intérêt pour les «cultural studies" qui ont taquiné en leur temps les milieux universitaires anglophones, nous ne pouvons pas tous nous reconnaitre dans le terme " culture », qui ne peut inclure, par exemple, les approches de type « sciences sociales ».

\section{Quel avenir pour le terme de « civilisation »?}

16 Il y a donc plusieurs raisons de garder et de promouvoir le terme "civilisation " pour décrire nos activités. Une raison positive évidente, tout d'abord, la résonnance du terme dans notre pays. Son caractère inclusif ensuite, qui permet de recouvrir tous les domaines, histoire, sciences politiques, économie, philosophie. Le terme est absolument le seul à pouvoir prétendre à cela. Enfin, son caractère distinctif, qui affirme clairement sa spécificité par rapport aux études littéraires. Pour revenir sur le contexte 
institutionnel de ce débat, il est donc essentiel que les postes soient fléchés "civilisation", si c'est dans ce domaine que sont les besoins des universités. La civilisation se décline ensuite de mille manières, et il est bien sûr possible aux collègues de valoriser leur spécialité, leur méthodologie ou leur champ de recherches en faisant preuve de créativité dans la signature de leurs œuvres ou de leurs messages.

\section{NOTES}

1. Centre de recherches en civilisation britannique

2. Il s'agit du réseau de recherches « l'Observatoire de la société britannique »

INDEX

Mots-clés : civilisation, disciplines, CRECIB

Keywords : discipline, interdisciplinarity, social sciences, CRECIB 